\title{
GI-MS48-P03 | MiCAPENROSE: A WORK OF ART AS A TOOL TO TEACH ANBD POPULARIZE CRYSTALLOGRAPHY
}

Ravy, Sylvain (Laboratoire de Physique des Solides, Orsay, FRA)

We present a recently created work of art, a Penrose tiling made of mica tiles sandwiched between two sheets of polarizers, and placed on a LED panel of $60 \mathrm{~cm} \times 60 \mathrm{~cm}$. Due to the birefringence of mica, the piece exhibits beautiful and shimmering interference colors, which changes as the viewer moves around. The irregularity of the muscovite mica gives different colors to the tiles themselves, enhancing the shimmering aspect of the piece. The frame is made in aluminium, reminiscent of quasi-crystals. The striking beauty of this artwork encourages the viewer to ask questions about the physical principles involved, the material it is made of, and the tiling it represents.

In this way, we can discuss: the nature of light and its polarization; the interference colors versus the rainbow colors; the birefringence of mica and its relation to its anisotropic, lamellar, atomic, crystalline structure; the chemical binding involved; the short-range ordered polymeric structure of the polarizer; the application of birefringence to mineralogy; the aperiodic long-range ordered crystalline structure represented by the Penrose tilling; the aluminium frame and the quasi-crystalline aluminium alloys. A future version of the piece will include a dislocation of the tiling, broadening the discussion to include the role of defects in solid state physics.

The poster, on which the piece will be installed, will detail all the pedagogical aspects which can be discussed from this very rich artwork.

[1] https://www.Ips.u-psud.fr/IMG/jpg/micapenrose 1.jpg?5065/2587a1feab58a0583b0e0a0bc5ddc818372b1f45 